\title{
Flexible boundary layer using exchange for embedding theories. I. Theory and implementation
}

\author{
Zhuofan Shen ${ }^{1,2}$ and William J. Glover ${ }^{1,2,3, a)}$ \\ 1) NYU Shanghai, 1555 Century Ave., Shanghai, 200122, China \\ ${ }^{2)}$ NYU-ECNU Center for Computational Chemistry at NYU Shanghai, 3663 Zhongshan Road North, Shanghai, \\ 200062, China \\ ${ }^{3)}$ Department of Chemistry, New York University, New York, NY, 10003, USA
}

Embedding theory is a powerful computational chemistry approach to exploring the electronic structure and dynamics of complex systems, with QM/MM being the prime example. A challenge arises when trying to apply embedding methodology to systems with diffusible particles, e.g. solvents, if some of them must be included in the QM region, for example in the description of solvent-supported electronic states or reactions involving proton transfer or charge-transfer-to-solvent: without a special treatment, inter-diffusion of QM and MM particles will lead eventually to a loss of QM/MM separation. We have developed a new method called Flexible Boundary Layer using Exchange (FlexiBLE) that solves the problem by adding a biasing potential to the system that maintains QM/MM separation. The method rigorously preserves ensemble averages by leveraging their invariance to exchange of identical particles. With a careful choice of the biasing potential, and the use of a tree algorithm to include only important QM and MM exchanges, we find the method has an MM-forcefield-like computational cost and thus adds negligible overhead to a QM/MM simulation. Furthermore, we show that molecular dynamics with the FlexiBLE bias conserves total energy and remarkably, dynamical quantities in the QM region are unaffected by the applied bias. FlexiBLE thus widens the range of chemistry that can be studied with embedding theory.

\section{INTRODUCTION}

A common strategy to modelling reactions in complex systems is to use an embedding approach, in which a chemically active region is treated with a high level of theory (e.g. ab initio electronic structure) and the remainder of the system is treated at a lower level of theory. The most widely used embedding method, and the subject of our first application, is QM/MM, which invokes a Quantum Mechanical (QM) treatment of the active region with the remainder of the system treated with Molecular Mechanics (MM) forcefields; ${ }^{1-21}$ however, QM-in-QM embedding has also seen significant interest in recent years. ${ }^{22-25}$

QM/MM has found broad applications ranging from enzymology, structural biology, materials science, and spectroscopy, among others. ${ }^{20,26-32}$ Another important use of $\mathrm{QM} / \mathrm{MM}$ is in describing solution-phase chemistry, ${ }^{28,33,34}$ since a solvent environment lends itself naturally to an MM description, with the solute treated at a QM level. This approach can be combined powerfully with ab initio molecular dynamics for a first-principles description of reactivity in complex systems. ${ }^{6,28,31,35,36}$ However, a problem arises when applying embedding theory to a system of diffusible particles, such as a solvent, if some of them are treated at the QM level: a QM/MM boundary must be made between identical molecules, and without special techniques, the QM and MM molecules will inter-diffuse, leading to a loss of QM/MM partitioning. Of course, one straightforward way to avoid this issue is to treat the solvent entirely at

\footnotetext{
a)Electronic mail: william.glover@nyu.edu
}

the MM level; however, this is not always possible, for example if the chemical process of interest involves proton or charge transfer with the solvent. Other solvated systems that pose a challenge for traditional QM/MM approaches are solvent-supported electronic states, a prime example of which are solvated electrons, which are excess electrons embedded in a liquid solvent. ${ }^{37-40}$

There are two broad classes of approaches to solving the boundary issue in $\mathrm{QM} / \mathrm{MM}$ with diffusible particles: Adaptive QM/MM and Constrained QM/MM. In the former approach, the treatment of particles changes dynamically between QM and MM descriptions as the molecule traverses the boundary. This is usually accomplished by an interpolation of QM and MM energies and/or forces between the regions, and several such methods have been developed, differing in how the boundary is defined and how the interpolation is achieved. ${ }^{3,7-9,11,14,16,18,21}$ Adaptive methods have the advantages that the number of QM molecules need not be conserved and diffusional dynamics are captured. However, it is known that current adaptive QM/MM methods suffer from structural and dynamical artefacts at the boundary due to a mismatch of QM and MM interactions, which must somehow be corrected. ${ }^{21}$ High quality MM forcefields, such as $\mathrm{MBP}$, show promise at overcoming this QM/MM mismatch issue. ${ }^{41}$

In contrast to Adaptive QM/MM, Constrained $\mathrm{QM} / \mathrm{MM}$ methods use a fixed definition of QM and MM atoms and apply some form of bias to the system to maintain their separation. Several methods fall under this category, including the method we develop below. To our knowledge, the first Constrained QM/MM method was Flexible Inner Region Ensemble Separator (FIRES), introduced by Rowley and Roux. ${ }^{10}$ FIRES adds a half- 
harmonic repulsion potential to MM molecules at the boundary, whose location dynamically adjusts based on the outermost QM molecule. The magnitude of bias required to maintain $\mathrm{QM} / \mathrm{MM}$ separation was however found to be large, so the authors cautioned against using FIRES with a large QM region or for the prediction of dynamical quantities. ${ }^{10}$ Shiga and Masia developed the Boundary based on Exchange Symmetry Theory (BEST) method which uses a carefully chosen bias potential to achieve QM/MM separation while preserving ensemble average quantities. ${ }^{12,13}$ However, similar to FIRES, a large magnitude of bias was required to maintain QM/MM separation, necessitating the use of small timesteps of 0.25 fs. These authors later developed Quasi-boundary based on Exchange Symmetry Theory (QUEST), which corrects for violations of exchange symmetry due to differences in the QM and MM potentials, at the expense of additional QM calculations on the exchanged particle configurations. ${ }^{42}$ They found these corrections improved the predictions of dynamical properties for a toy system compared to BEST; however, a QM/MM implemention of QUEST has not yet been described. The Boundary Constraint with Correction (BCC) method, developed by Takahashi and co-workers, applies a postprocessing correction to constrained QM/MM simulations to remove effects of the bias from equilibrium properties, when the bias potential itself is not constructed to preserve equilibrium properties; ${ }^{19}$ however, no correction for dynamical properties was developed. Based on the current status of constrained QM/MM methods, it is evident that there is room for improvement, in particular with respect to reducing the magnitude of required bias so that dynamical quantities are minimally affected, at least in the inner QM region and for sub-diffusional timescales.

Building on the formalism introduced in BEST, ${ }^{12}$ in this work we develop the Flexible Boundary Layer using Exchange (FlexiBLE) method. The key idea is a construction of the biasing force that acts, in principle, on every QM and MM pair and all their possible combinatorial exchanges. With a careful choice of biasing potential, however, the biasing forces can be truncated outside a narrow boundary layer of QM and MM molecules, and the surviving terms can be efficiently enumerated with a tree algorithm. As a result, the influence of the bias is highly localized to the boundary region, and we demonstrate that ensemble and even dynamical properties are preserved in benchmark one-electron mixed/quantum classical (MQC) simulations of the aqueous solvated electron, $e_{(\mathrm{aq})}^{-}{ }^{43,44}$ In the companion paper, we use FlexiBLE to build an ab initio many-electron QM/MM model of $e_{(\mathrm{aq})}^{-}$to explore its structural and electronic properties.

The remainder of the paper is as follows. In section II A, we briefly review the formalism of BEST to provide context for a description of the FlexiBLE method, which is introduced in section II B. In section III, we benchmark the method on equilibrium and dynamical properties of a MQC description of the hydrated electron. Finally, conclusions are drawn in Section IV.

\section{THEORY}

\section{A. BEST}

To provide the context for our development of FlexiBLE, we first review the formalism of BEST introduced by Shiga and Masia. ${ }^{12}$ The starting point is the recognition that an ensemble average is invariant to exchanges of like particles. Thus, by treating QM and MM solvent particles as identical (necessarily an approximation unless the MM potential perfectly mimics the QM potential), one can perform a weighted ensemble average wherein configurations that have MM and QM mixing (exchanges) are included with a low weight, whereas configurations that have MM and QM separation are included with high weight. In particular, consider the canonical ensemble average of observable $X$ :

$$
\langle X\rangle=\frac{\int X(\mathbf{R}) \exp (-\beta V(\mathbf{R})) d \mathbf{R}}{\int \exp (-\beta V(\mathbf{R})) d \mathbf{R}},
$$

where $V(\mathbf{R})$ is the system's total potential energy, $\beta=$ $1 / k_{\mathrm{B}} T$ is the inverse of the temperature multiplied by Boltzmann's constant, and $\mathbf{R}=\left(\cdots, \mathbf{R}_{i}, \cdots, \mathbf{R}_{j}, \cdots\right)$ is a configuration of atomic positions. In Eq. 1, configurations with an exchange of QM and MM particles are averaged with equal weights. We could instead add their contributions to the ensemble average with different weights by introducing a normalized bias function with the following properties:

$$
\begin{gathered}
f_{i j k \cdots} \equiv f\left(\cdots, \mathbf{R}_{i}, \cdots, \mathbf{R}_{j}, \cdots, \mathbf{R}_{k}, \cdots\right) \geq 0 \\
\sum_{L} \hat{P}_{L}\left(f_{i j k \cdots}\right)=1,
\end{gathered}
$$

where $\hat{P}_{L}$ is an operator that permutes the indices $i j k \cdots$ and the second equality above ensures that $f$ is normalized after summing over all possible QM and MM exchanges. Shiga and Masia showed that with a bias function that satisfies Eqs. 2 and 3, and using the permutational symmetry of $X$ and $V$, the ensemble average is formally unaffected by the inclusion of the bias function: ${ }^{12}$

$$
\begin{aligned}
\langle X\rangle & =\frac{\int X \exp (-\beta V) f_{i j k \ldots d \mathbf{R}}}{\int \exp (-\beta V) f_{i j k \ldots d \mathbf{R}}} \\
& =\frac{\int X \exp (-\beta \tilde{V}) d \mathbf{R}}{\int \exp (-\beta \tilde{V}) d \mathbf{R}}
\end{aligned}
$$

where the second equality results from incorporating the bias function with an added bias potential:

$$
\begin{aligned}
\tilde{V}_{i j k \cdots} & =V+V_{i j k \cdots}^{\mathrm{bias}} \\
V_{i j k \cdots}^{\mathrm{bias}} & =-k_{\mathrm{B}} T \log f_{i j k \cdots}
\end{aligned}
$$


To ensure normalization, the bias function can be written as:

$$
f_{i j k \cdots}=\frac{h_{i j k \cdots}}{\sum_{L} \hat{P}_{L}\left(h_{i j k \cdots}\right)}
$$

where $h_{i j k \ldots}$ is an unnormalized penalty function, and the denominator of Eq. 8 is a normalization factor that sums the penalty function over all possible QM and MM particle exchanges, resulting in $\left(N_{\mathrm{QM}}+N_{\mathrm{MM}}\right) ! /\left(N_{\mathrm{QM}} ! N_{\mathrm{MM}} !\right)$ unique terms.

Eq. 5 holds true for any bias function satisfying Eqs. 2 and 8 , and this provides a great deal of flexibility in the choice of penalty, $h$, in Eq. 8, which we will take advantage of below in our formulation of FlexiBLE. To maintain QM/MM separation, $h$ should be non-zero for configurations with $\mathrm{QM} / \mathrm{MM}$ separation and vanishingly small for configurations with QM/MM mixing. In this work, following Shiga and Masia, ${ }^{12}$ we take the QM region to be a sphere centered around the origin of the system, with MM particles outside the sphere. Generalizations to other QM geometries are possible. ${ }^{12}$ Perfect QM/MM separation is then achieved when the radial distance from the origin of every QM particle is less than the radial distance of every MM particle. Shiga and Masia proposed the following penalty function to bias the ensemble towards QM/MM separation: ${ }^{12}$

$$
\begin{aligned}
h_{i j \cdots, k l \cdots} & =\prod_{m=i, j, \cdots}^{N_{Q M}} \prod_{n=k, l, \cdots}^{N_{M M}} g_{m n} \\
g_{i k} & = \begin{cases}1, & x_{i}<x_{k} \\
\exp \left(-\alpha\left(x_{i}-x_{k}\right)\right), & x_{i} \geq x_{k},\end{cases}
\end{aligned}
$$

where $x_{i}$ and $x_{k}$ are the radial distances from the origin of the QM and MM particles respectively, and $g_{i k}$ is a pair function of $\mathrm{QM}$ and $\mathrm{MM}$ distances that is unity for QM particles closer to the origin than MM particles (thus favoring QM/MM separation), and decays exponentially to zero with the distance of any QM particle further from the origin than an MM particle (thus biasing against QM/MM mixing). The exponent parameter, $\alpha$, controls the rate of decay of bias with QM-MM distance: larger values of $\alpha$ achieve better QM/MM separation, although as we show below, at the expense of requiring a small MD timestep.

While Eq. 9 formally applies a biasing force to every QM and MM particle, and therefore one might worry that their dynamics are strongly influenced by the bias, the product-of-exponentials form of the penalty function means that for a large enough $\alpha$, the dominant bias forces will be imposed only on a single pair corresponding to the furthest QM and closest MM particles. This motivated Shiga and Masia to develop a Single Exchange (SE) approximation to the bias function, wherein Eq. 9 involves only the pair function between the furthest QM and closest MM particles, which after normalization via
Eq. 8 leads to the following bias function: ${ }^{12,13}$

$$
\begin{aligned}
f_{i, k}^{\mathrm{SE}} & =\frac{g_{i k}}{g_{i k}+g_{k i}} \\
& =\frac{1}{1+\exp \left(+\alpha\left(x_{i}-x_{k}\right)\right)},
\end{aligned}
$$

where $i$ and $k$ index the furthest QM and closest MM particles respectively.

The pair function and resulting bias potential of BEST in the SE approximation are shown as the black and red curves respectively in Fig. 1(a). Here it is seen that in the limit of large QM and MM separation, the bias potential tends to a linear increasing function of distance, meaning a constant bias force is applied to the furthest QM and closest MM particles to restore QM/MM separation. The magnitude of the restoring force is proportional to $\alpha$, which explains why BEST in the SE approximation can fail to maintain $\mathrm{QM} / \mathrm{MM}$ separation for small $\alpha{ }^{12}$
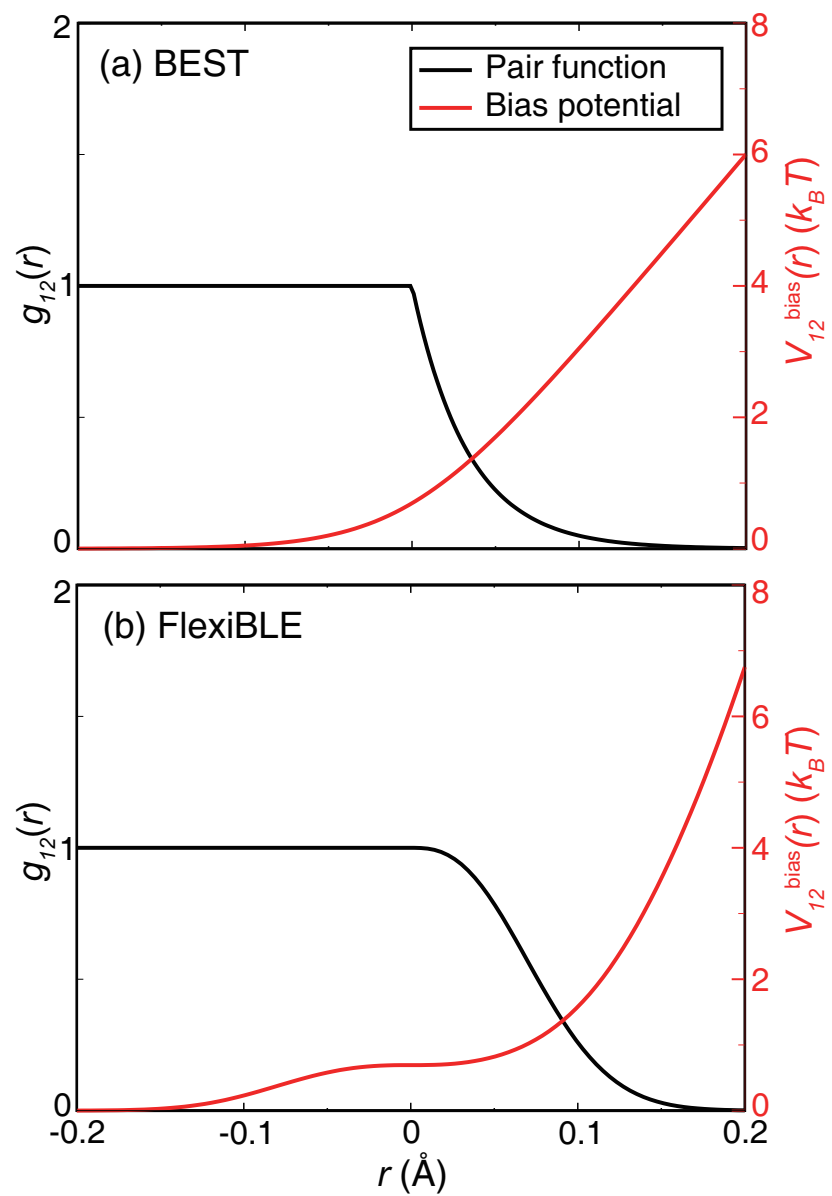

FIG. 1. Pair functions (black curves, left axis) and bias potentials (red curves, right axis) for a single QM and MM pair as a function of their displacement difference, $\Delta x=x_{i}-x_{k}$. Panel (a) BEST (Eq. 10) with $\alpha=30 \AA^{-1}$, panel (b) Flexi$\operatorname{BLE}$ (Eq. 14) with $\alpha=15 \AA^{-1}$.

The SE approximation has the advantage that it avoids the formally factorially scaling number of exchanges re- 
quired by Eq. 8, and the approximation can be made better by increasing the value of $\alpha$ in Eq. 11. However, while Eq. 11 is an analytic function for a given QM and MM pair, since it is defined in terms of the furthest QM and closest MM particles, whose identities can change discontinuously in the course of a dynamics simulation, $f^{\mathrm{SE}}$ is a non-analytic function of the particles coordinates, and therefore using it as a biasing function in molecular dynamics leads to energy non-conservation, as we show below.

A step up in complexity from the SE approximation is the Double Exchange (DE) approximation, which considers pair functions involving the furthest two QM and closest two MM particles. ${ }^{12}$ Then, following Eq. 9, the penalty function becomes the product of four pair functions:

$$
h_{i j, k l}=g_{i k} \times g_{i l} \times g_{j k} \times g_{j l},
$$

and the bias function is normalized by a sum of $(2+$ $2) ! /(2 ! \times 2 !)=6$ terms:

$$
f_{i j, k l}^{\mathrm{DE}}=\frac{h_{i j, k l}}{h_{i j, k l}+h_{k j, i l}+h_{l j, k i}+h_{i k, j l}+h_{i l, k j}+h_{k l, i j}},
$$

where $i j$ and $k l$ index the two furthest QM and two closest MM particles respectively. While the DE approximation might be expected to improve over the SE approximation, we find below that it exhibits poorer energy conservation than the SE approximation. This results from the derivative discontinuity of the pair function in Eq. 10 at $x_{i}=x_{k}$, shown as the kink in the black curve in Fig. 1(a).

The issues of derivative discontinuities in the BEST bias potential motivated us to explore alternative functional forms for the pair potential, and led to the development of the FlexiBLE method described in the next section.

\section{B. FlexiBLE}

Our FlexiBLE method involves two key differences to BEST. The first modification we make is to the pair function, $g_{i k}$, to ensure it applies an increasing bias force with QM and MM distance while also having continuous first and second derivatives. The second modification is to include all non-negligible permuted penalty functions, $h_{i j \cdots, k l \ldots}$, by truncating the denominator of the bias function in Eq. 8 using a tree algorithm. We provide details of these developments below.

\section{Pair function}

As noted above, the BEST pair function, $g_{i k}$, in Eq. 10 leads to a constant bias force (being proportional to the derivative of the pair function) between a pair of QM and MM particles with large QM and MM separation (see
Fig. 1). The magnitude of the bias force that restores QM and MM separation is furthermore proportional to the exponent parameter, $\alpha$, so if this parameter is too small, QM and MM separation is not maintained (see for example Fig. 4 of Ref. 12). Although this issue can be resolved by choosing a sufficiently large value of $\alpha$, we find below that the resulting large bias forces require a small timestep of 0.25 fs for a stable MD simulation, and this incurs a significant computational overhead unless a multiple timestep algorithm is used. ${ }^{13}$ To allow the use of a smaller $\alpha$ parameter, it would be preferable to have a bias force that increases with QM and MM separation. This can be achieved by replacing the exponent in Eq. 10 with a function that has a quadratic decay at large QM and MM separation. Although a simple half-Gaussian function for $x_{i} \geq x_{k}$ would satisfy this property, the second derivative of such a pair function would be discontinuous at $x_{i}=x_{k}$ and this would likely cause convergence problems in geometry optimizations. After some experimentation, we opted for the following rational form of the pair function's exponent:

$$
g_{i k}^{\text {FlexiBLE }}= \begin{cases}1, & x_{i}<x_{k} \\ \exp \left(-\frac{\alpha^{3}\left(x_{i}-x_{k}\right)^{3}}{1+\alpha\left(x_{i}-x_{k}\right)}\right), & x_{i} \geq x_{k}\end{cases}
$$

The FlexiBLE pair function of Eq. 14 and the resulting bias potential for a pair of QM and MM particles are shown Fig. 1(b), where we see our choice of a rational function exponent yields a smooth pair function (continuous first and second derivatives) while also exhibiting a quadratically increasing bias potential in the limit of large QM and MM separation.

It is interesting to explore the behavior of the bias potential for a pair of QM and MM particles with $\Delta x=$ $x_{i}-x_{k} \leq 0$. In this regime, the QM and MM particles are not mixed, yet the bias potential is non-zero. This results from the normalization condition on the bias function (Eq. 8): as QM and MM particles approach each other from $\Delta x<0$, the bias function with exchanged $\mathrm{QM}$ and $\mathrm{MM}$ indices becomes non-negligible, so the magnitude of the bias function is reduced from unity. Contributions to the bias potential from QM and MM pairs that have $x_{i} \leq x_{k}$ must therefore be considered; however, the bias potential is seen to decay rapidly to zero for $x_{i} \ll x_{k}$ suggesting a truncation of terms involving exchanges between QM and MM particles with large negative displacement differences is possible. Furthermore, the rapid increase of $V^{\text {bias }}$ with $x_{i} \gg x_{k}$ strongly biases against a large number of QM and MM pairs having $\Delta x>0$, which always contribute to the bias potential, again suggesting a truncation of terms is possible. Indeed, we show below that the FlexiBLE bias function can be aggressively truncated. Nevertheless, the form of the bias function does permit a small degree of QM/MM mixing around $\Delta x=0$ close to the QM/MM boundary. This is true for any choice of pair function, including the form used in BEST. The width of this mixing region is controlled by how rapidly the pair function decays with 
QM/MM separation, and can thus be made negligibly small with a suitably large value of $\alpha$.

\section{Truncating the bias function}

The second modification to BEST we make is to include all non-negligible QM and MM exchanges in the calculation of the denominator of the bias function in Eq. 8. Since the total number of exchanges increases factorially as $\left(N_{\mathrm{QM}}+N_{\mathrm{MM}}\right) ! /\left(N_{\mathrm{QM}} ! N_{\mathrm{MM}} !\right)$, which would introduce a computational bottleneck for $\mathrm{QM}$ regions of more than a few particles, to make FlexiBLE practical, we must aggressively truncate the sum over exchanges in Eq. 8. The key to making this possible is a product of pair function form of the penalty function, which we retain from the BEST formalism:

$$
h_{i^{\prime \prime} j^{\prime \prime} \cdots, k^{\prime \prime} l^{\prime \prime} \ldots}^{\text {FlexiBLE }}=\prod_{m=i^{\prime \prime}, j^{\prime \prime}, \cdots}^{N_{\mathrm{QM}}^{\mathrm{imp}}} \prod_{n=k^{\prime \prime}, l^{\prime \prime}, \ldots}^{N_{\mathrm{MM}}^{\mathrm{imp}}} g_{I_{m} I_{n}}^{\text {FlexiBLE }},
$$

where $i^{\prime \prime}, j^{\prime \prime}, \cdots$ index important QM particles (to be defined below) of which there are $N_{\mathrm{QM}}^{\mathrm{imp}}$, and $k^{\prime \prime}, l^{\prime \prime}, \cdots$ index important MM particles of which there are $N_{\mathrm{MM}}^{\mathrm{imp}}$. $I_{m}$ is the original index of the $m$-th important particle. Since the bias potential favors QM/MM separation, most of the QM and MM pairs give $g_{m n}^{\text {FlexiBLE }}=1$ and their contributions therefore do not need to be included in the penalty function. Furthermore, given the exponential form of the pair function in Eq. 14, the magnitude of the penalty function in Eq. 15 is dominated by the smallest value of $g_{m n}^{\text {FlexiBLE }}$, which comes from the pair involving the furthest QM and closest MM particles after a particular exchange pattern. When the distance difference between the furthest QM and closest MM particle is large following exchange, the value of $g_{m n}^{\text {FlexiBLE}}$, and therefore also $h^{\text {FlexiBLE }}$, for that exchange pattern can be safely neglected from the denominator of Eq. 8.

The observations above imply two levels of truncation in forming the bias function: Firstly, most QM and MM particles do not contribute to the denominator of the bias potential, because they give $g_{m n}^{\text {FlexiBLE }}=1$ (and therefore could be neglected in the evaluation of Eq. 15) and $g_{m n}^{\text {FlexiBLE }} \approx 0$ when exchanged (and therefore yield a value of $h^{\text {FlexiBLE }}$ that can be neglected from the denominator of Eq. 8). Secondly, most exchange patterns in the denominator of Eq. 8 can be neglected because they involve multiple exchanges of QM and MM particles that result in a negligibly small $h^{\text {FlexiBLE }}$. The denominator of the bias function can therefore be aggressively truncated and the FlexiBLE bias function is thus:

$$
f^{\text {FlexiBLE }}=\frac{h_{i j \cdots, k l \cdots}^{\text {FlexiBLE }}}{\sum_{L \in \mathbf{L}^{\text {imp }}} \hat{P}_{L}\left(h_{i^{\prime \prime} j^{\prime \prime} \cdots, k^{\prime \prime} l^{\prime \prime} \ldots}^{\text {FlexiBLE }}\right)},
$$

where $\mathbf{L}^{\mathrm{imp}}$ is the set of important QM/MM exchanges which lead to a non-negligible contribution to the denominator, i.e. for which $\hat{P}_{L}\left(h_{i^{\prime \prime} j^{\prime \prime} \ldots, k^{\prime \prime} l^{\prime \prime} \ldots}\right)>h_{\text {thre }}$, where $h_{\text {thre }}$ is a threshold parameter. Note: the numerator penalty function is evaluated for all QM and MM pairs, without truncation (indicated by unprimed indices). This is because the numerator could have a value below the threshold parameter, but must not be approximated as zero, or else the bias potential would diverge.

The first type of truncation of the FlexiBLE bias function involves a pre-screening of important QM and MM atoms that have at least one exchange resulting in a nonnegligible value of $h^{\text {FlexiBLE }}>h_{\text {thre }}$ in the denominator of Eq. 16. To find the important QM and MM particles we start by reordering their indices based on the particle's distance from the QM origin. Note: this reordering might involve exchanges of $\mathrm{QM}$ and $\mathrm{MM}$ particles, but as explained below, this is an exchange pattern that will always be retained in the denominator of Eq. 16. Then, as we show in Appendix C, a rigorous upper bound to $h^{\text {FlexiBLE }}$ resulting from any exchange pattern (following reordering) that exchanges the $p^{\prime}$ th particle from QM to $\mathrm{MM}$, and no closer QM particles, is given by the value of the ordered penalty function with a single exchange of the $p^{\prime}$ th particle with the innermost MM particle (with index $\left.k^{\prime}\right)$, i.e.:

$$
h_{p^{\prime}}^{\mathrm{QM} \text { bound }}=h_{i^{\prime} j^{\prime} \cdots k^{\prime} \cdots, p^{\prime} l^{\prime} \cdots}^{\mathrm{FlexiBL}},
$$

where the primed indices indicate they have been reordered. Likewise, an upper bound to $h^{\text {FlexiBLE }}$ resulting from any exchange pattern that exchanges the $q^{\prime}$ th particle from MM to QM, and no further MM particles, is given by its exchange with the outermost QM particle (having index $N^{\mathrm{QM}}$ ):

$$
h_{q^{\prime}}^{\mathrm{MM} \text { bound }}=h_{i^{\prime} j^{\prime} \cdots q^{\prime}, k^{\prime} l^{\prime} \cdots N^{\mathrm{QM}} \ldots}^{\mathrm{FlexiBL}} .
$$

Furthermore, it is clear that the bounds decay monotonically with increasing distance from the QM/MM boundary, so that important $\mathrm{QM}$ and $\mathrm{MM}$ atoms can be quickly pre-screened to satisfy $h_{p^{\prime}}^{\mathrm{QM}}$ bound $>h_{\text {thre }}$ and $h_{q^{\prime}}^{\mathrm{MM} \text { bound }}>h_{\text {thre }}$.

The ability to pre-screen important QM and MM particles demonstrates that the FlexiBLE bias potential incurs non-negligible bias forces only on $\mathrm{QM}$ and $\mathrm{MM}$ particles within a thin layer at the QM/MM boundary. The boundary layer's radius depends on the instantaneous location of the outermost QM and innermost MM particles (following reordering), and the width of the layer depends on the bounds in Eq. 17 \& 18. The boundary layer is therefore flexible and allows for density fluctuations in both the QM, MM, and their boundary regions. A schematic of the boundary layer is shown in Fig. 2.

While pre-screening the important QM and MM particles is vital to an efficient implementation of FlexiBLE, used alone it does not avoid a steeply scaling computational cost with number of QM particles (confirmed numerically in Fig. 9 below). This can be understood from Fig. 2: as the total number of QM particles grows, so too 


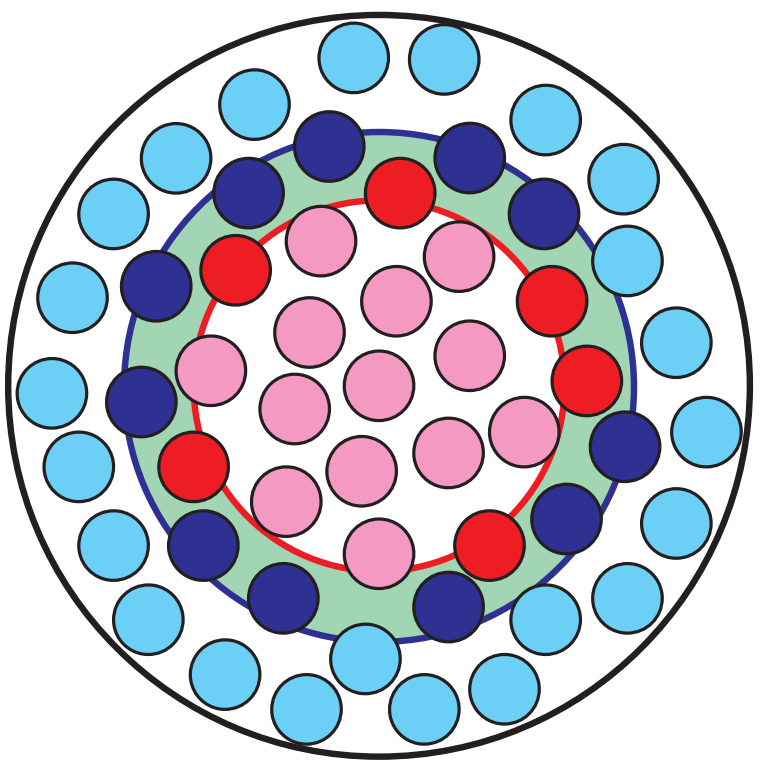

FIG. 2. Schematic of the FlexiBLE boundary layer. Particles are indicated by small circles (QM: red shade, MM: blue shade). Important QM and MM particles that experience bias forces are indicated by dark-shaded colors and form a boundary layer (shaded in green).

does the number of important particles in the boundary layer, which scales as the ratio of the QM region's surface area to its volume $\left(N^{\mathrm{QM}}\right)^{2 / 3}$ for the spherical $\mathrm{QM} / \mathrm{MM}$ partitioning of interest. Thus, to reduce the scaling, a second type of truncation must be performed: to neglect small values of the exchanged penalty function, $\hat{P}_{L}\left(h_{i^{\prime \prime} j^{\prime \prime} \ldots, k^{\prime \prime} l^{\prime \prime} \ldots}\right)$ in the denominator of Eq. 16, which we achieve with a tree algorithm.

We initiate the tree by noting that the one exchange pattern of $\mathrm{QM}$ and $\mathrm{MM}$ indices that is guaranteed to have the largest contribution to the denominator of the bias function is the exchange that results in an ordering of $\mathrm{QM}$ and $\mathrm{MM}$ distances, i.e. perfect $\mathrm{QM} / \mathrm{MM}$ separation, with a penalty function equal to 1 . This exchange pattern must always be included, and since the penalty function from all other exchange patterns must be less than or equal to the ordered penalty function, we take this to be the root of the tree structure. The tree is then traversed as follows: taking each node on the previous level as a parent, we generate child nodes on the next level by performing a single exchange between each QM particle and the next immediate outer particle if it is an MM particle. We avoid duplication of children with each other by comparison against a growing list of visited child nodes on each level. Without truncation, the tree would terminate on a single node corresponding to complete MM and QM exchange, although of course this would ordinarily correspond to a negligibly small penalty function, and the tree could have been truncated many levels before this.

We generate the tree in the above way for two reasons: 1) without any truncation, the procedure is guaranteed to visit every unique $\mathrm{QM}$ and $\mathrm{MM}$ exchange, as proved in Appendix A. 2) The penalty functions for child nodes constructed as a single exchange between a QM and the next outer particle (if MM) are guaranteed to be less than or equal to the penalty function of their parent node, as we prove in Appendix B. Thus, once a parent node is below the penalty function threshold, $h_{\text {thre }}$, no children need to be generated and that branch of the tree can be truncated: this is the key to avoiding strict factorial scaling in the number of denominator terms.

In Fig. 3, we show a schematic of our tree algorithm applied to a hypothetical system of 3 important QM and 3 important MM particles. As in this example, the zeroth level always contains a single root node corresponding to the ordered indices. In addition, the root node always gives rise to a single child on the first level corresponding to an exchange of the outermost QM and innermost MM particle indices (3 and 4 respectively in this example). If the displacement difference between the outermost QM and innermost MM particle was sufficiently large compared to $\alpha^{-1}$, the tree could be truncated at this level (we do find this to occur occasionally during the MD trajectories described below). In the hypothetical example considered in Fig. 3 however, this is not the case, and the second level thus has two children from exchanging QM particle 2 with MM particle 3, and QM particle 4 with MM particle 5. These nodes then generate three children on level 3 (note, one child is shared between the two parents of level 2). At this level, our algorithm detects that the node corresponding to $h_{126,345}^{\text {FlexiBE }}$ falls below the threshold (indicated by the change of color to red), and therefore no children are generated from this node. At level 4, only a single child meets the threshold criterion, while at level 5 no children are above the threshold, and therefore the algorithm stops. All surviving nodes are summed to give the denominator of Eq. 16: note, we include parent nodes that were below threshold, since we had already computed their bias function; however, their children are discarded. We provide pseudocode for the tree algorithm in the supporting information. We verify numerically below that the number of surviving child nodes exhibits sub-exponential scaling with QM size, with a lower exponent power than without truncation.

While the tree algorithm described above is an efficient means to sum over important exchanges of the penalty function in the denominator of the FlexiBLE bias function, the relation between the penalty function threshold, $h_{\text {thre }}$, and the error introduced by truncation of the denominator is not immediately clear. In particular, since the penalty function is always positive, truncation necessarily leads to an underestimate of the true denominator. Furthermore, the number of neglected nodes in the tree grows factorially with the number of important QM and MM particles following pre-screening. While our tree algorithm guarantees that each neglected node has a penalty function below the threshold, one might be concerned that an astronomical number of neglected nodes 


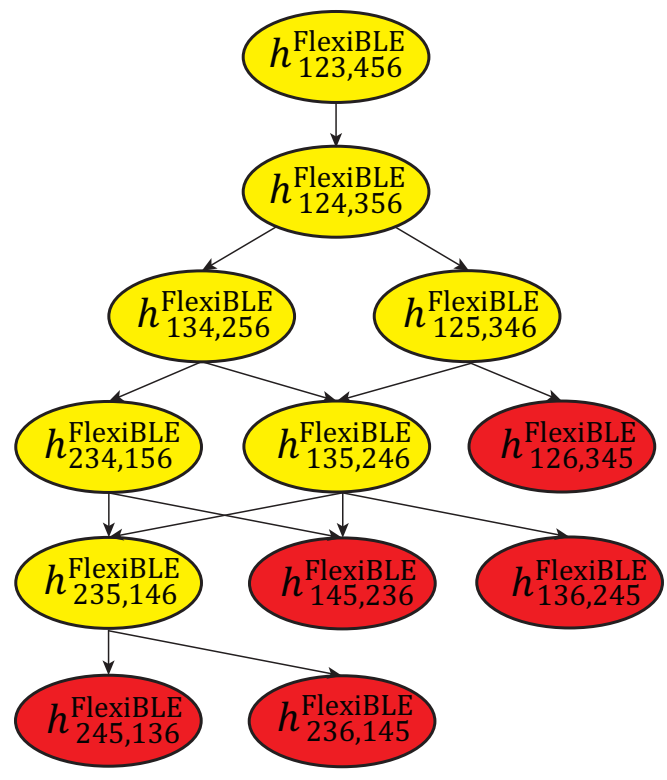

FIG. 3. The FlexiBLE tree algorithm applied to a hypothetical system of 3 important QM and 3 important MM particles. Each node of the tree (ellipses) indicates a unique QM/MM exchange pattern (shown by the indices of $h^{\text {FlexiBLE }}$ ). Child nodes are generated by performing a single exchange between each QM particle and the next immediate outer particle if it is an MM particle, indicated by arrows. Duplicate children are not double counted. Yellow nodes indicate the bias function is above the threshold, $h^{\text {FlexiBLE }}>h_{\text {thre }}$, and further children are generated. Red nodes indicate $h^{\text {FlexiBLE }} \leq h_{\text {thre }}$ and no children are generated (i.e. the branch is truncated). The tree is terminated when all nodes on a level are below threshold, which happens here on level $5 . h^{\text {FlexiBLE }}$ is summed over all surviving nodes to give the denominator of Eq. 16.

sum to a non-negligible value. To allay this concern, we use an adaptive thresholding, by sweeping through the tree in an iterative fashion, and at each new iteration, tightening the threshold, $h_{\text {thre }}$, to 0.5 of its previous value. The relative change in the denominator of Eq. 16 is monitored between iterations, and when below a convergence parameter, $\gamma$, the iterations stop. Given the logarithmic dependence of the bias potential on the bias function (Eq. 7), $\gamma$ directly provides an estimate of the error in the bias potential due to truncation, in units of $k_{\mathrm{B}} T$. We find a value of $\gamma=0.001 k_{\mathrm{B}} T$ yields stable MD trajectories, and convergence is reached typically within two or three FlexiBLE iterations, suggesting that the error in the denominator is typically no worse than an order of magnitude larger than $h_{\text {thre }}$.

Putting all the pieces together, we present pseudocode for FlexiBLE in Algorithm 1 below. Note: analytical bias forces on the QM and MM particles follow straightforwardly from chain-rule derivatives of the pair, penalty, bias, and potential functions (Eqs. 7, 14-16) with respect to particle coordinates. We thus evaluate and accumulate bias force contributions simultaneously with the compu- tation of each surviving $h^{\text {FlexiBLE }}$ term. With the prescreening of important $\mathrm{QM}$ and $\mathrm{MM}$ pairs, combined with the tree-algorithm enumeration of important QM and MM exchanges, evaluation of the bias potential and forces has an MM-forcefield-like computational cost, and thus adds negligible overhead to a $\mathrm{QM} / \mathrm{MM}$ simulation.

\section{RESULTS AND DISCUSSION}

To benchmark the FlexiBLE method, we consider the quintessential system exhibiting solvent-supported electronic states: the hydrated electron, $e_{(\mathrm{aq})}^{-}$, which corresponds to an excess electron embedded in liquid water. We choose an MQC description of the system such that all water molecules are identically treated at an MM level. We can then divide the water molecules into inner and outer regions (MM* and MM respectively), allowing us to verify the accuracy of FlexiBLE without possible errors arising due to a mismatch of QM and MM interactions, and allowing a direct comparison of structural and dynamical properties against results from MQC simulations without FlexiBLE partitioning. The low computational cost of MQC also allows us to reach very large MM* sizes and hundreds of picoseconds of sampling.

\section{A. Computational details}

We used a $20 \AA$ radius spherical droplet model of the condensed-phase $e_{(\mathrm{aq})}^{-}$, containing 1035 water molecules, with an excess electron solvated at the center of the droplet. Although FlexiBLE is fully compatible with Periodic Boundary Conditions (PBC), we chose a spherical droplet model to match the many-electron FlexiBLE$\mathrm{QM} / \mathrm{MM}$ model of $e^{-}(\mathrm{aq})$ that we develop in the companion paper. ${ }^{45}$ Comparing our droplet results against previous PBC MQC results shows the impact on both structural and dynamic quantities from using a spherical droplet model to be minimal.

An initial water droplet configuration was generated from a previous $\mathrm{PBC}$ MQC simulation ${ }^{46}$ of the hydrated electron with the Turi-Borgis (TB) potential ${ }^{44}$ by first centering the electron at the origin, tiling space with periodic replicas of the cubic simulation cell, then pruning to form a spherical droplet of the closest 1035 water molecules based on oxygen distance from the origin. This number of water molecules was chosen to be consistent with the output of a SolvateCap command of the tleap program in Amber18 for a water droplet radius of $20 \AA .^{47}$ To prevent water evaporation into the surrounding vacuum, a half-harmonic confining potential was applied to the oxygen atoms: $V^{\text {conf }}=0.5 k^{\text {conf }}\left(r_{\mathrm{O}}-r_{\text {drop }}\right)^{2} \Theta\left(r_{\mathrm{O}}-\right.$ $\left.r_{\text {drop }}\right)$, where $r_{\mathrm{O}}$ is the distance of the oxygen atom from the origin, taken to be the center of mass of the system, $r_{\text {drop }}=20 \AA$ is the droplet radius, $k^{\text {conf }}=10 \mathrm{eV} / \AA^{2}$ is the confining force constant, and $\Theta(r)$ is the Heaviside function. 


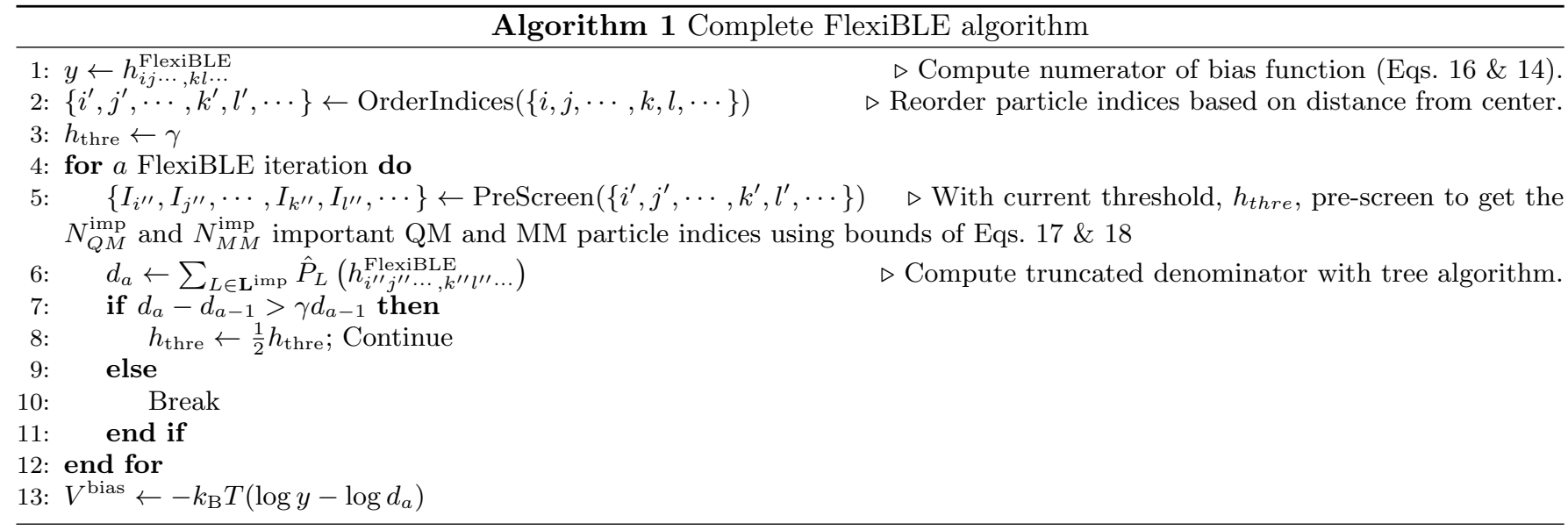

To be consistent with previous PBC MQC simulations, ${ }^{46,48}$ the $\mathrm{SPC} /$ Flex water model was used and the excess electron's wavefunction was discretized in a Fourier-Grid (FG) basis and the one-electron Schrodinger Equation was solved with an iterative diagonalization method (further details below). For Ground-State (GS) simulations, we used $14 \times 14 \times 14$ grid points with a spacing of $1.1034 \AA$. For excited-state calculations, the grid was extended to $32 \times 32 \times 32$, keeping the grid spacing the same. Since the FG did not span the entire simulation cell, following Ref. 49, we periodically shifted particle positions by an integer number of grid spacings in order to recenter the electron's wavefunction, and avoid FG boundary artefacts. Furthermore, a harmonic restraint with a force constant of $k^{\mathrm{e}-\mathrm{COM}}=10 \mathrm{eV} / \AA^{2}$ was placed on the electron centroid using quantum-biased $\mathrm{MD}^{46,50}$ in order to tether the electron to the center of the droplet, and prevent it from diffusing to the droplet surface. Coulomb and Lennard-Jones pair interactions were not truncated.

For each system (unbiased, BEST, FlexiBLE), we computed observables from ten trajectories of $50 \mathrm{ps}$ in length. The initial configurations of each trajectory were sampled at 5 ps intervals from another trajectory of 50 ps in length, following an equilibration period of $10 \mathrm{ps}$. Initial velocities were randomly sampled from the MaxwellBoltzmann distribution, using a different random number seed for each trajectory. Temperature was maintained at $298 \mathrm{~K}$ using the Bussi-Parrinello thermostat ${ }^{51}$ with a friction coefficient of $0.5 \mathrm{ps}^{-1}$. Unless otherwise stated, the velocity Verlet algorithm with a timestep of 0.5 fs was used to propagate MD. ${ }^{52}$

At each MD timestep, the lowest eigenvalue of the one-electron Hamiltonian with the TB potential was solved iteratively using the Generalized Davidson (GD) algorithm, ${ }^{53}$ as implemented in SLEPc 3.14.1. ${ }^{54}$ Forces on the water molecules from the excess electron were evaluated with the Hellmann-Feynman theorem, ${ }^{55}$ which is exact for a FG basis. To analyze energy-gap fluctuations, the lowest five electronic excited states of $e_{(\mathrm{aq})}^{-}$ were computed in an ex post facto fashion on snap- shots taken from the GS MQC trajectories. However, for these excited-state calculations, we found that the GD algorithm occasionally had convergence problems and would miss roots, so we instead used the Krylov-Schur algorithm $^{56}$ along with a second-order Chebyshev polynomial spectral transformation of the eigenspectrum, described previously. ${ }^{49}$

Unless otherwise stated, 64 inner water molecules were treated as "QM" particles using either BEST or FlexiBLE; however, since these particles retained MM forcefields, we label them $\mathrm{MM}^{*}$ to avoid confusion. $\mathrm{MM}^{*}$ and MM particle distances from the origin, needed in Eqs. 10 and Eqs. 14, were computed based on the water oxygen distance from the center of mass of the entire system. This choice maintained translation invariance of the Hamiltonian.

\section{B. Structural properties}

\section{BEST}

We start by exploring how accurate BEST is for ensemble averaged structural properties of $e_{(\mathrm{aq})}^{-}$. The quantity of interest is the Radial Distribution Function (RDF), $g(r)$, of the water atoms relative to the excess electron centroid position. Fig. 4 plots the RDF for $e^{-}$-oxygen distances from BEST simulations in the SE and DE approximations (solid red and dashed blue curves of panel (a) respectively). Here we used a high value of the exponent parameter, $\alpha=189 \AA$ to match the previous BEST work. $^{12,13}$ With this choice, we found that a timestep of 0.25 fs was needed for stable MD propagation. Results without a MM*/MM biasing potential are labelled "Full system" and are shown as purple circles.

From Fig. 4, we see overall good agreement between BEST and Full system results except for noticeable artefacts at a radius of $7.5 \AA$, corresponding to the $\mathrm{MM}^{*} / \mathrm{MM}$ transition region. Interestingly, BEST-DE performs worse than SE. Evidence that derivative discontinuities in the BEST potential (discussed in Section 


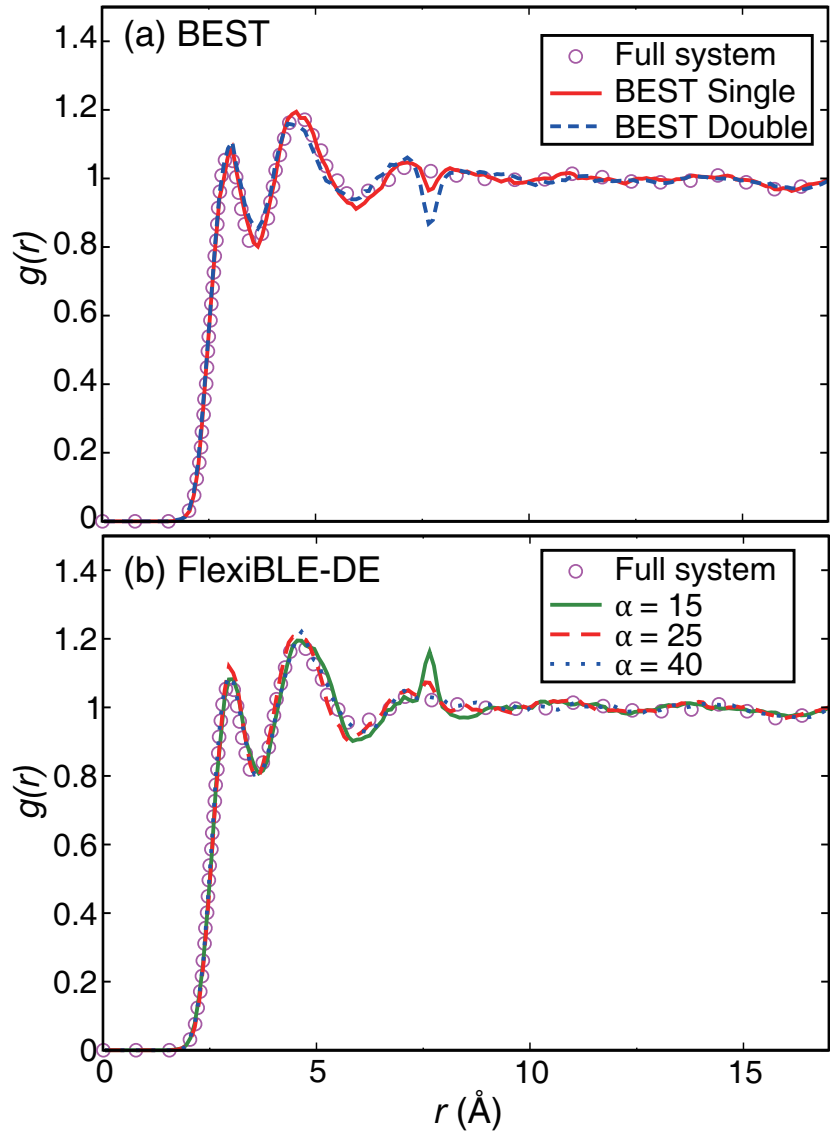

FIG. 4. RDF of $e^{-}$-oxygen distances from constrained $\mathrm{MM}^{*} / \mathrm{MM}$ simulations with 64 inner $\mathrm{MM}^{*}$ water molecules. Panel (a): BEST using the single exchange or double exchange approximations (solid red and dashed blue curves respectively) with $\alpha=189 \AA^{-1}$. Panel (b): FlexiBLE-DE (double exchange) with $\alpha=15,25,40 \AA^{-1}$ (solid green, dashed red, and blue dotted curves respectively). Unconstrained "Full system" results are indicated as purple circles.

II A) are the source of the problem is seen in Fig. 5, which reveals noticeable drifts in the total energy of $\sim 0.8 \mathrm{eV} / \mathrm{ps}$ for BEST-SE (red curve) and worsening to $\sim 1.7 \mathrm{eV} / \mathrm{ps}$ for BEST-DE (blue curve). We note that these problems were not observed in the original BEST work, ${ }^{12,13}$ which however used much smaller QM (or $\mathrm{MM}^{*}$ ) regions of 12 or fewer water molecules, compared to 64 in this work. In Fig. S1, we verify that BEST-SE conserves energy and reproduces full system results with $\mathrm{MM}^{*}=4$. The derivative discontinuity problems therefore seem to become appreciable only for large QM regions. This makes sense, since a large QM region will experience frequent exchanges between the furthest QM and second furthest QM particle, leading to derivative discontinuities resulting from the SE approximation. The DE approximation also apparently fails to ameliorate this problem, since the pair function it relies on (Eq. 10) itself has a derivative discontinuity, which is not perfectly cancelled out, unlike in the SE approximation.

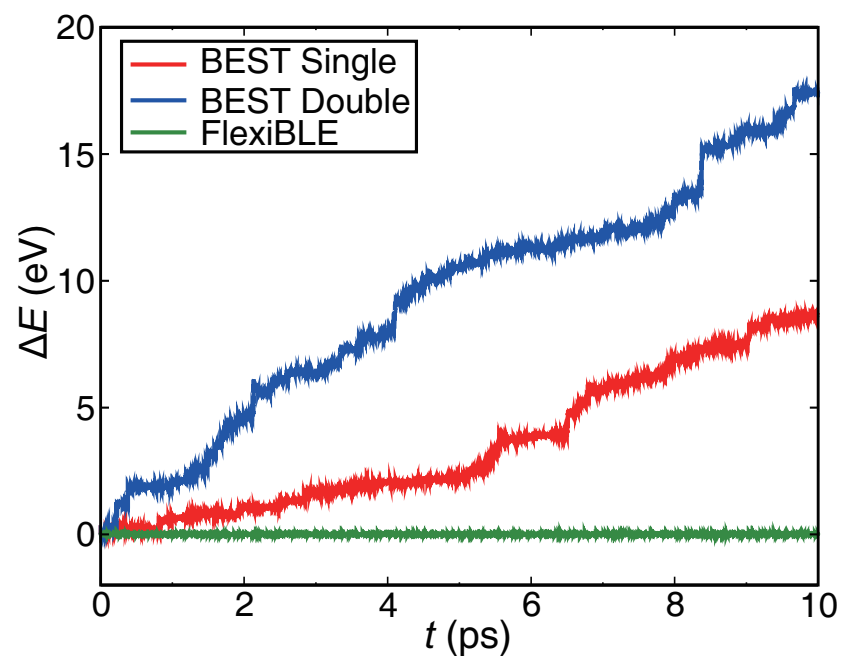

FIG. 5. Total energy drift along a 10-ps trajectory for BEST-SE, BEST-DE, and FlexiBLE simulations (red, blue, and green curves respectively) in the NVE ensemble with a timestep of 0.25 fs. The BEST simulations used $\alpha=189 \AA^{-1}$, and FlexiBLE used $\alpha=15 \AA^{-1}$.

The lack of energy conservation in BEST leads to a temperature gradient, with the $\mathrm{MM}^{*}$ region heating up relative to the MM region, as shown in Fig. 6. This is despite the use of a thermostat, and the resulting nonequilibrium condition explains why ensemble quantities like the RDF in Fig. 4 do not agree with full system results. It is possible that the temperature gradients in BEST could be ameliorated with a more aggressive thermostating, in particular by associating an independent thermostat with each particle, and increasing the friction coefficient from our choice of $0.5 \mathrm{ps}^{-1}$. However, such an approach would strongly affect dynamical quantities, which we wish to preserve.

\section{FlexiBLE}

We now turn to the performance of FlexiBLE in describing the RDF of $e_{(\mathrm{aq})}^{-}$. We consider first FlexiBLE within a double exchange approximation (FlexiBLE-DE), i.e. with a bias function of Eq. 13, but using the FlexiBLE pair and penalty functions of Eqs. 14 and 15. As Fig. 4(b) shows, when the exponent parameter is chosen to be too small, such as $\alpha=15 \AA$, FlexiBLE-DE also exhibits artefacts in the RDF at the boundary region, indicating the DE appoximation breaks down for small $\alpha$. However, as the exponent parameter is increased to $\alpha=25 \AA$, agreement with Full system results (purple circles) improves, and perfect agreement is seen for $\alpha=40$ $\AA$. This shows that the FlexiBLE pair function in Eq. 14 is one of the key improvements to BEST, and allows for large QM regions to be studied.

While FlexiBLE-DE is a useful method, our finding that a large exponent parameter of $\alpha=40 \AA$ and 


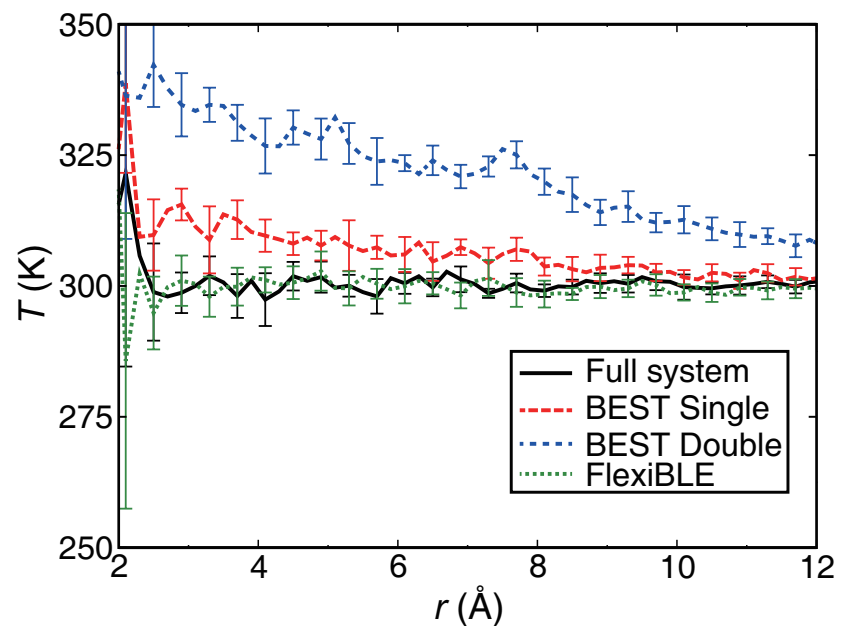

FIG. 6. Temperature distribution for BEST-SE, BEST-DE and FlexiBLE simulations (red long dashed, blue dashed, and green dotted curves respectively) as a function of distance from the excess electron. Full system results are shown as the solid black curve. A noticeable temperature gradient is observed for the BEST simulations, while FlexiBLE maintains thermal equilibrium, in agreement with the full system results.

therefore a small timestep of 0.25 fs are needed means that the method comes at significant computational expense, since without the FlexiBLE-DE biasing potential, a timestep of 0.5 fs would be acceptable for liquid water. This motivated us to develop the FlexiBLE method described in Section IIB, i.e. retaining all important QM/MM exchanges according to Eq. 16.

RDFs from FlexiBLE simulations using $\alpha=15 \AA$ and a timestep of 0.5 fs are shown in Fig. 7, where we see perfect agreement between FlexiBLE and Full system results for both $e^{-}-\mathrm{O}$ and $e^{-}-\mathrm{H}$ RDF in panels (a) and (b) respectively. This explains the failure of BEST-DE for small $\alpha$ (as seen in Fig. 4): exchanges beyond only the furthest two $\mathrm{MM}^{*}$ and closest two MM particles must be retained. FlexiBLE recovers all the important $\mathrm{MM}^{*} / \mathrm{MM}$ exchanges in a computationally efficient manner. In fact, as we show in the Supporting Information, FlexiBLE allows for an even smaller $\alpha=5 \AA$ and a timestep of 1.0 fs, while still maintaining QM/MM separation.

We further show a decomposition of the RDF into MM* and MM contributions (solid red and blue shaded regions respectively in Fig. 7), which demonstrates that FlexiBLE successfully maintains $\mathrm{MM}^{*} / \mathrm{MM}$ separation. The small amount of overlap between $\mathrm{MM}^{*}$ and MM contributions to the $e^{-}-\mathrm{O}$ RDF in panel (a) largely results from density fluctuations that dynamically move the location of the $\mathrm{MM}^{*} / \mathrm{MM}$ boundary, resulting in a smearing out of their distributions in the ensemble average. The $\mathrm{MM}^{*}$ and $\mathrm{MM} e^{-}-\mathrm{H}$ distributions in panel (b) display more overlap than the $e^{-}-\mathrm{O}$ distributions since the bias potential is applied to the oxygen atom only, i.e. some MM water molecules can rotate their hydrogen
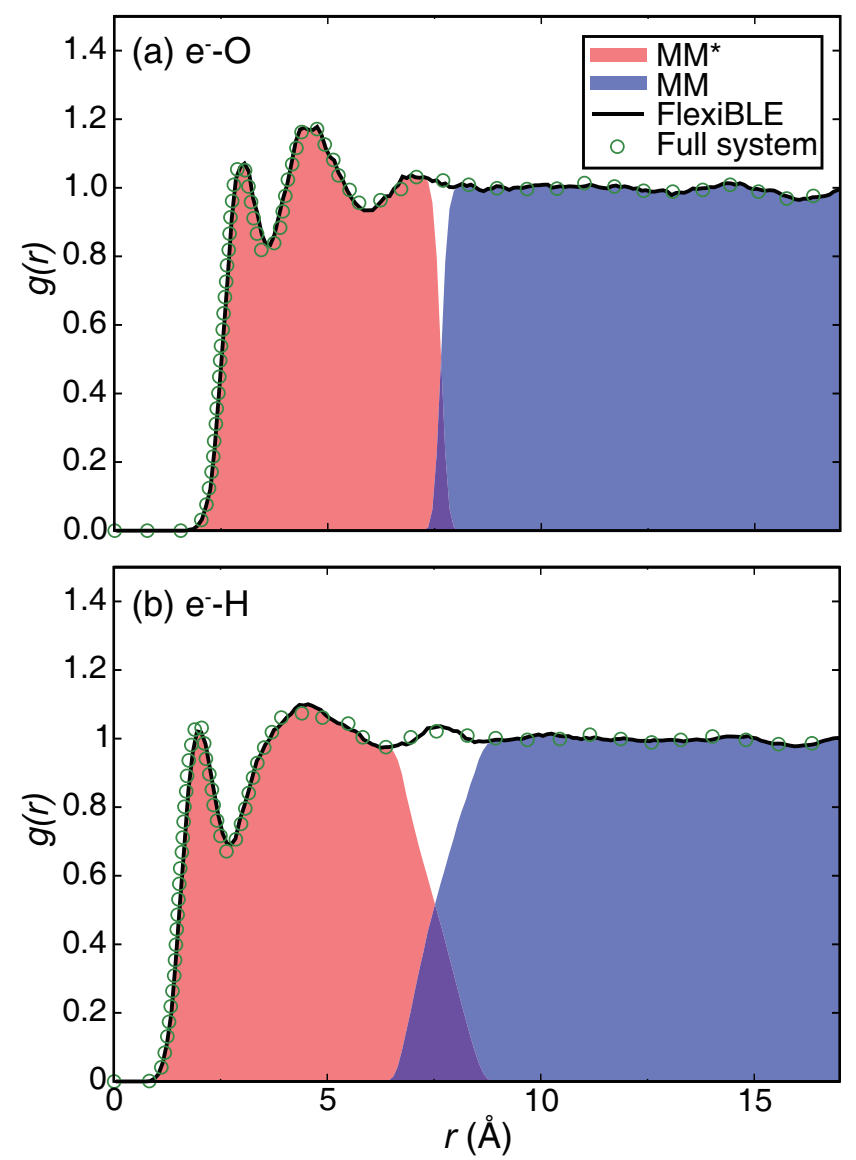

FIG. 7. RDF of $e_{(\mathrm{aq})}^{-}$from FlexiBLE simulations with $\alpha=15$ $\AA^{-1}$ (solid black curves) compared to unbiased Full system simulations (green circles). Panel (a): $e^{-}$-Oxygen distributions. Panel (b): $e^{-}$-Hydrogen distributions. Also shown is a breakdown into $\mathrm{MM}^{*}$ (red) and MM (blue) contributions. FlexiBLE reproduces full system results and successfully maintains $\mathrm{MM}^{*} / \mathrm{MM}$ separation.

atoms to be within the $\mathrm{MM}^{*}$ region. This is expected and desirable behavior: applying orientational constraints on MM particles would lead to an artificial structuring at the boundary, so we apply boundary constraints only on a single atom (or virtual site) of each $\mathrm{MM}^{*}$ and $\mathrm{MM}$ molecule. We confirm that FlexiBLE perfectly conserves energy (green curve of Fig. 5) and does not exhibit temperature gradients (green curve of Fig. 6).

\section{Dynamical properties}

Having seen that FlexiBLE successfully reproduces structural properties of $e_{(\mathrm{aq})}^{-}$compared to simulations without a bias, we next consider dynamical properties. Of particular interest is the energy gap autocorrelation function, since this reports the dynamical couplings between the solvent and QM solute, and can be used to predict the absorption spectrum of $e_{(\mathrm{aq})}^{-}$, including nu- 
clear quantum effects. ${ }^{57}$ The gap correlation function for state $i$ is defined as

$$
C_{i}(t)=\frac{\left\langle\delta E_{i}(t) \cdot \delta E_{i}(0)\right\rangle}{\left\langle\delta E_{i}(0)^{2}\right\rangle},
$$

where $\delta E_{i}(t)=\left(E_{i}(t)-E_{0}(t)\right)-\left\langle E_{i}-E_{0}\right\rangle$, and $E_{i}$ is the adiabatic energy of state $i$ ( 0 indexes the ground state).

Fig. 8 shows $C(t)$ for the first and fifth excited states (panels a and b respectively). Full system results (green dot dashed curves) agree with previously published PBC MQC simulation results using the TB potential, ${ }^{57}$ where we see that both state 1 and 5 exhibit rapid decorrelation, reaching $\sim 10 \%$ of their initial values by $t=500$ fs. Fourier transforms of the correlation functions reveal that the energy gaps of either state are predominantly coupled to translational and librational motions of water; however, the gap to state 5 exhibits stronger coupling to higher frequency vibrational modes. ${ }^{57}$ The difference between the gap correlation functions for state 1 and 5 results from the cavity localized nature of the ground state and state 1 , so that their energies are modulated in a fairly parallel fashion by first-solvent-shell intramolecular motion. On the other hand, state 5 is delocalized and weakly coupled to solvent motions, such that its energy gap fluctuations are dominated by the ground-state energy, which is more strongly coupled to vibrations of the first solvent shell.

We test FlexiBLE's ability to reproduce dynamical quantities by first considering a very small $\mathrm{MM}^{*}$ region of 2 water molecules. This choice places the $\mathrm{MM}^{*} / \mathrm{MM}$ partition in the first solvent shell of $e_{(\mathrm{aq})}^{-}$and would be expected to alter its dynamics. Indeed, we see noticeable deviations between the correlation functions from FlexiBLE 2MM* (dotted black curve) and full-system results (dot-dashed green curve), although the differences are modest. In particular, the gap correlation functions for both state 1 and state 5 decay slower for FlexiBLE $2 \mathrm{MM}^{*}$, presumably because first solvent shell translations and librations are hindered by the biasing potential. Remarkably however, upon extending FlexiBLE to include $16 \mathrm{MM}^{*}$ molecules (two solvation shells), the computed gap correlation functions (solid red curves) are indistinguishable from full system results. Similar agreement is found also for larger $\mathrm{MM}^{*}$ regions.

The electronic structure of $e_{(\mathrm{aq})}^{-}$is strongly coupled to solvent motions (being a solvent-supported species), particularly via translational and librational modes that should be most strongly affected by the FlexiBLE potential, so the fact that we do not observe any detectable influence of the bias potential on the dynamics of $e_{(\mathrm{aq})}^{-}$gives us confidence the technology could be used to study the dynamics of many other solutes at a FlexiBLE-QM/MM level, providing a sufficiently large QM region is chosen.

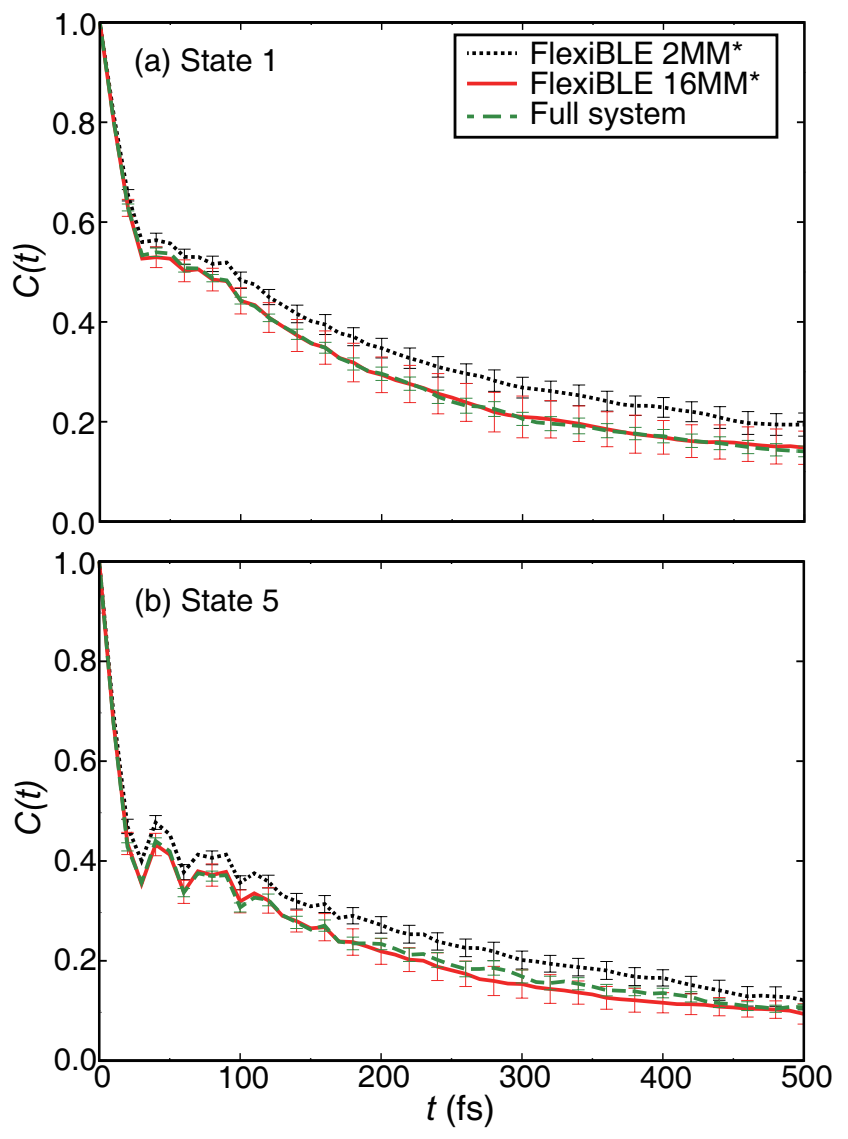

FIG. 8. Gap Correlation Function, $C(t)$, for $e_{(\mathrm{aq})}^{-}$from MQC simulations with FlexiBLE $\mathrm{MM}^{*} / \mathrm{MM}$ partitioning. Panel (a): ground to State 1 energy gap correlation function. Panel (b): ground to State 5 energy gap correlation function. Results are shown with two different sized $\mathrm{MM}^{*}$ regions: $2 \mathrm{MM}^{*}$ (dotted black curves) and $16 \mathrm{MM}^{*}$ (solid red curves), with the latter in excellent agreement with full system results (dotdashed green curves).

\section{Computational scaling}

We finally consider the computational scaling of FlexiBLE. Without truncation, the cost of the method is expected to scale as the number of penalty function terms in the denominator of Eq. 16, $N_{\text {terms }}$, so we consider this quantity first. As described in Section II B, there are two stages of truncation: first a pre-screening of important $\mathrm{MM}^{*}$ and MM particles, then a truncation of exchanges between the surviving important $\mathrm{MM}^{*}$ and MM particles in the denominator of Eq. 16. We considered the scaling of $N_{\text {terms }}$ following both of these truncations by performing a series of $e_{(\text {aq })}^{-}$simulations with the number of $\mathrm{MM}^{*}$ waters varied from 4 to 384 . An exponent parameter of $\alpha=15 \AA^{-1}$ was used for all simulation. 50 ps of dynamics was propagated and $N_{\text {terms }}$ was averaged along the trajectory. All simulations were performed on a single core of an Intel Xeon E5-2630 v4 2.20GHz chip.

In Fig. 9 we plot how $N_{\text {terms }}$ scales with the size of the 
QM region, taken here to be the number of $\mathrm{MM}^{*}$ water molecules. The black curve shows the total number of exchanges after a pre-screening of important $\mathrm{MM}^{*}$ and $\mathrm{MM}$ particles. As expected based on theoretical grounds (see Supporting Information), following pre-screening, $N_{\text {terms }}$ scales as $2^{a\left(N^{\mathrm{QM}}\right)^{\frac{2}{3}}}$, where $a$ is a free parameter. The relatively high power of $\left(N^{\mathrm{QM}}\right)^{\frac{2}{3}}$ means that for $\mathrm{MM}^{*}$ regions of greater than $\sim 200$ QM particles, the FlexiBLE boundary potential evaluation would become the most computationally expensive part of the MQC calculation. This motivates the truncation of $\mathrm{QM} / \mathrm{MM}$ exchanges with our tree algorithm (dashed red curve), which we see dramatically reduces $N_{\text {terms }}$ and lowers their scaling to $\sim 2^{b\left(N^{\mathrm{QM}}\right)^{0.53}}$, with $b$ a free parameter.

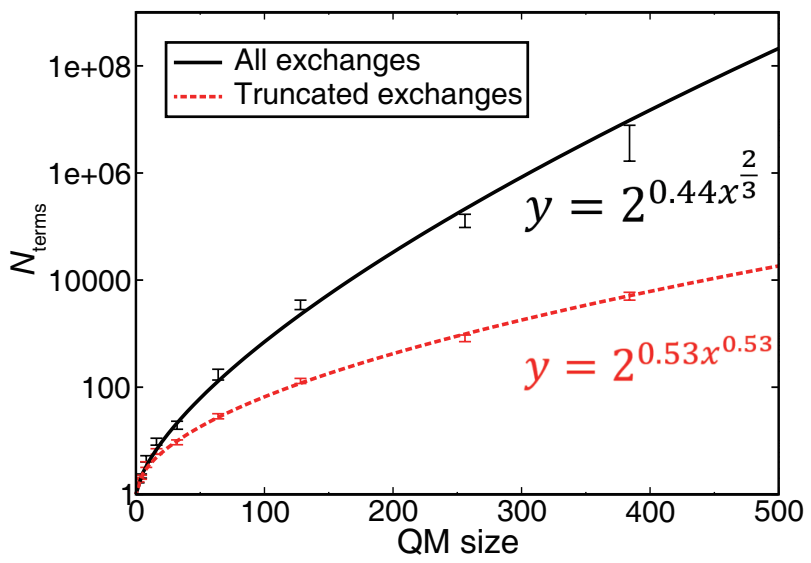

FIG. 9. Scaling of number of FlexiBLE denominator terms (Eq. 16), $N_{\text {terms }}$ with QM size. The effects of two types of truncation are shown: the black curve shows the number of terms following a pre-screening of important QM and MM particles. The dashed red curve shows the number of terms following truncation of the denominator according to the FlexiBLE tree algorithm. Equations of best fit are shown.

The computational cost of FlexiBLE is explored in Fig. 10, which plots the average CPU time per timestep spent on FlexiBLE and its breakdown into different operations. The first operation is to sort QM and MM indices (red squares): this is seen to have a low cost that is constant with QM size, since the cost of sorting depends on the total number of particles, $N_{\mathrm{QM}}+N_{\mathrm{MM}}$, which is fixed in our simulations. The next operation is the pre-screening of important QM and MM labels (black circles). This also has a low cost that fits to a sublinear power law with QM size. The evaluation of the FlexiBLE numerator penalty function in Eq. 16 (purple upward triangles) also has a negligible cost that does not scale with QM size. The two operations that dominate a FlexiBLE calculation are thus the evaluation of denominator penalty function terms (turquoise downward triangles), with a scaling that matches Fig. 9, and the bookkeeping associated with maintaining a growing child-node history to avoid their duplication (blue diamonds). Since both of these operations happen in the inner loop of the Flexi-
BLE algorithm, they exhibit similar sub-exponential scaling. As we see, it is the computations associated with the child history that dominate the total cost of FlexiBLE.

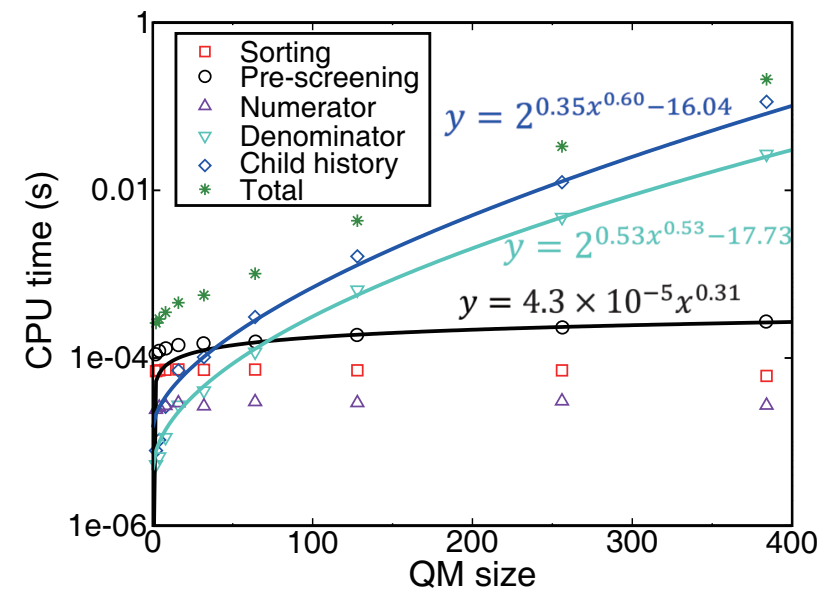

FIG. 10. Computational cost of FlexiBLE with varying QM size, and its breakdown into different contributions, in order of operation. CPU times report averages per timestep. Red squares: cost associated with sorting particle indices by distance from center. Black circles: pre-screening of important QM and MM indices. Purple upward triangles: numerator of Eq. 16. Turquoise downward triangles: denominator of Eq. 16. Blue diamonds: bookkeeping of child history to avoid duplication of denominator terms.

That the bookkeeping associated with avoiding child node duplication in the FlexiBLE algorithm should dominate the calculation is a testament to how significantly our truncation algorithm has reduced the number of surviving denominator penalty function terms: without truncation, they would overwhelm the computational cost. Maintaining a child history is the remaining expensive operation since at every level of the tree, each new child must be compared against a growing list of previously visited children on that level, and the number of children grows steeply with the depth of the tree, even with truncation. We have made this operation as efficient as possible by using a binary representation of the QM and MM labels of each node. As a result, the observed overall sub-exponential scaling of FlexiBLE has a sufficiently small prefactor that the method has a CPU time of $<1 \mathrm{~s}$ even for $\mathrm{QM}$ sizes of $\sim 400$ atoms, which is certainly negligible compared to the cost of $a b$ initio electronic structure calculations on QM regions of this size. Nevertheless, the sub-exponential scaling of FlexiBLE, compared to the polynomial scaling of electronic structure, means that a crossover point will occur for QM regions of sufficiently large size, after which the computational cost of FlexiBLE would dominate. The crossover point depends on the level of electronic structure, but appears to be in the thousands of QM particles, for which AIMD is already infeasible, without considering the cost of FlexiBLE, and this will likely remain true for the foreseeable future. Thus, we consider FlexiBLE to be prac- 
tical for some time to come.

\section{CONCLUSIONS}

In this paper, we developed a new constrained QM/MM method called FlexiBLE, which allows for a QM/MM partitioning between identical diffusible particles such as a solvent or gas. We demonstrated that the method maintains QM/MM separation while rigorously preserving ensemble averaged quantities. FlexiBLE improves on the BEST constrained QM/MM method ${ }^{12}$ in several ways: 1) by a careful choice of pair function (Eq. 14) that has a quadratic exponent for large distance, QM/MM separation is maintained even for small bias exponents of $\alpha=5 \AA$, allowing for MD timesteps of $1.0 \mathrm{fs}$, compared to 0.25 fs in BEST. 2) with the new pair function, combined with a tree-algorithm enumeration of all important QM and MM exchanges, FlexiBLE conserves total energy, and does not display temperature gradients as seen in BEST, even for large QM sizes of hundreds of molecules. 3) Our choice of pair function in Eq. 14 leads to bias forces that are small in magnitude per particle, yet highly localized to a narrow layer of QM and MM particles close to the boundary. As a result, the bias forces minimally perturb the dynamics of the system and we demonstrated that FlexiBLE reproduces full system energy gap time correlation functions of $e_{(\mathrm{aq})}^{-}$, providing that at least two solvation shells are included in the QM region.

We expect that FlexiBLE embedding will be broadly useful in simulating static and dynamic equilibrium properties of complex systems. As a first showcase of the method, in the companion paper, ${ }^{45}$ we apply FlexiBLE to the dynamics and electronic structure of $e_{(\mathrm{aq})}^{-}$at a many-electron QM/MM level. Although the initial focus of the method has been on solvated systems, the bias function can be easily modified to describe surface geometries, allowing a description of heterogeneous interfaces. Furthermore, since the FlexiBLE bias potential is a function of nuclear coordinates only, and has an MMforcefield-like computational cost, the approach is entirely compatible with other embedding schemes, such as polarizable, ${ }^{58-61}$ density, ${ }^{22,23}$ and mean-field embedding theories. ${ }^{24}$ We thus believe that constrained QM/MM partitioning, as in FlexiBLE, is a promising alternative to adaptive QM/MM.

\section{SUPPLEMENTARY MATERIAL}

See the supplementary material for further details on our tree algorithm, the computational scaling of FlexiBLE, a comparison of BEST with small versus large QM regions, BEST versus FlexiBLE energy conservation, FlexiBLE with a small bias exponent, and FlexiBLE with a large QM region.

\section{ACKNOWLEDGMENTS}

This work was supported by the National Natural Science Foundation of China Young Scientist Fund (Grant No. 21603145), the Science and Technology Commission of Shanghai Municipality Foreign Experts Program (Grant No. 21WZ2503600), the NYU-ECNU Center for Computational Chemistry, and start-up funds from NYU Shanghai. We thank Siyao Guo for invaluable assistance with the proof in Appendix A.

\section{DATA AVAILABILITY}

The data that support the findings of this study are available within the article and its supplementary material. Molecular coordinates are available from the corresponding author upon reasonable request.

\section{Appendix A: Proof that tree visits all QM/MM exchanges}

Here we prove that all QM/MM exchanges are visited by the tree algorithm described in Section II B. We start by summarizing our algorithm in the language of graph theory.

- With the set of important particle indices, $U:=$ $\left\{1, \ldots, N_{\mathrm{QM}}^{\mathrm{imp}}+N_{\mathrm{MM}}^{\mathrm{imp}}\right\}$, we consider the directed graph $G=\left(\left\{h_{S_{0}}, \ldots, h_{S}, \ldots\right\}, E\right)$, where $S_{0}:=$ $\left\{1, \ldots, N_{\mathrm{QM}}^{\mathrm{imp}}\right\}$ and for every set $S \subseteq[U]$ of size $N_{\mathrm{QM}}^{\mathrm{imp}}$, we define the node $h_{S}$ to represent $h_{S, U \backslash S}^{\mathrm{FlexiBLE}}$.

- There exists a directed edge from $h_{S}$ to $h_{S^{\prime}}$ if $S^{\prime}$ is obtained by removing some $a \in S$ from $S$ and adding the corresponding $a+1 \notin S$ into $S$.

Claim. There exists a direct path from $h_{S_{0}}$ to every node in the graph.

Proof. For every $h_{S}$, let $d_{S}$ denote the difference between the sum of elements in $S$ and the sum of elements in $S_{0}$ (i.e. $d_{S}$ is the level of the tree). We will prove the claim inductively according to $d_{S}$. The base is true because $S$ must be $S_{0}$ itself when $d_{S}=0$.

Assume that for integer $n \geq 0$ there exists a directed path from $h_{S_{0}}$ to any $h_{S}$ with $d_{S}=n$. Now we prove that $h_{S^{\prime}}$ can be reached for any $S^{\prime}$ with $d_{S^{\prime}}=n+1$. It suffices to show that there exists a directed edge between $S^{\prime}$ and $S$ for some $S$ with $d_{S}=n$. Then we can have a path from $S_{0}$ to $S^{\prime}$ by extending the path from $S_{0}$ to $S$ via this edge.

Let $a_{1}<\cdots<a_{N_{\mathrm{QM}}^{\mathrm{imp}}}$ denote sorted elements in $S^{\prime}$ and $b_{1}<\cdots b_{N_{\mathrm{MM}}^{\mathrm{imp}}}$ denote sorted elements in $U \backslash S^{\prime}$. Observe that $b_{1}<a_{N_{\mathrm{QM}}^{\text {imp }}}$ (otherwise $S^{\prime}$ can only be $S_{0}$ and $d_{S^{\prime}}=$ $0)$. Then there must exist $i, j$ such that $a_{i}=b_{j}+1 .^{62}$ Hence, by swapping $a_{i}$ and $b_{j}$, we will obtain the desired $S$ with $d_{S}=n$. 


\section{Appendix B: Proof of tree hierarchy}

To simplify the proofs of inequalities involving the FlexiBLE penalty function, $h^{\text {FlexiBLE }}$ (Eq. 15), we first introduce a new notation to identify the QM and MM particle indices that the penalty function depends on. To start, we assume that all QM and MM particles have been ordered by distance from the center of the QM region, as discussed in Section II B. A QM/MM list used to build $h^{\text {FlexiBLE }}$ is then defined as $[\mathrm{AA} \cdots \mathrm{ABB} \cdots \mathrm{B}]$ where $\mathrm{A}$ and $\mathrm{B}$ are $\mathrm{QM}$ and $\mathrm{MM}$ particle labels respectively, and the location of the label in the list indicates the particle index. Perfect QM/MM separation corresponds to the situation where all A labels are on the left of B labels. Each unique list therefore corresponds to a unique $h^{\text {FlexiBLE }}$ function. For example, a list of $[\mathrm{AABABB}]$ corresponds to $h_{124,356}^{\mathrm{FlexiBLE}}$.

By the definitions of Eq. 14 and 15, the root of the FlexiBLE tree, $h_{\text {root }}^{\text {FlexiBLE}}$, which has perfect QM/MM separation, is:

$$
h_{\text {root }}^{\text {FlexiBLE }}=h[\mathrm{AA} \cdots \mathrm{ABB} \cdots \mathrm{B}]=1 .
$$

$h_{\text {root }}^{\text {FlexiBLE }}$ is the largest term in the denominator because all of its pair functions, $g_{m n}$, are 1 .

According to the tree algorithm discussed in Section IIB, we define a child node by performing on its parent a single exchange between a QM particle and the next immediate outer particle if it is an MM particle. Thus, the only child generated from the root node is $h[\mathrm{AA} \cdots \mathrm{ABABB} \cdots \mathrm{B}]$, with a value of $g_{N_{\mathrm{QM}}, N_{\mathrm{QM}}+1} \leq 1$. Thus we see that the first-level node of the tree is guaranteed to be equal to or less than the zeroth-level node.

To show that the hierarchical nature of the tree holds generally for any parent and child, consider a parent node of $[\cdots \mathrm{AB} \cdots]$, where $\mathrm{A}$ is the $i$ th particle and $\mathrm{B}$ is the $(i+1)$ th particle. Suppose that there are $N$ B labels to the left of $i$ with indices $\left\{n_{1}, n_{2}, \ldots, n_{N}\right\}$ and $M$ A labels to the right of $i+1$, with indices $\left\{m_{1}, m_{2}, \ldots, m_{M}\right\}$. Note: there can be an arbitrary number of A labels to the left of $i$ and an arbitrary number of B labels to the right of $i+1$. Upon exchange of $i$ and $i+1$ labels, the relation between the value of the child node and parent node is:

$$
h_{\text {child }}^{\text {FlexiBLE }}=h_{\text {parent }}^{\text {FlexiBLE }} \times g_{i+1, i} \times \frac{g_{n_{1}, i+1}}{g_{n_{1}, i}} \times \frac{g_{n_{2}, i+1}}{g_{n_{2}, i}} \times \cdots \times \frac{g_{n_{N}, i+1}}{g_{n_{N}, i}} \times \frac{g_{i, m_{1}}}{g_{i+1, m_{1}}} \times \frac{g_{i, m_{2}}}{g_{i+1, m_{2}}} \times \cdots \times \frac{g_{i, m_{M}}}{g_{i+1, m_{M}}},
$$

where we have retained only non-unit terms. Since $g_{m n}$ is monotonically decreasing from 1 to 0 , we have the following relations for any exchange:

$$
\begin{aligned}
g_{i+1, i} & \leq 1, \\
g_{n_{a}, i+1} & \leq g_{n_{a}, i}, \forall n_{a}, \\
g_{i, m_{b}} & \leq g_{i+1, m_{b}}, \forall m_{b},
\end{aligned}
$$

which together with Eq. B2 proves that any child node has a value equal to or less than its parent:

$$
h_{\text {child }}^{\text {FlexiBLE }} \leq h_{\text {parent }}^{\text {FlexiBLE }} .
$$

\section{Appendix C: Proof of FlexiBLE penalty function bounds}

As discussed in section II B, the number of $h^{\text {FlexiBLE }}$ terms can be aggressively truncated by including only the important QM/MM particles that always contribute at least one pair function $g^{\text {FlexiBLE }}$ (Eq. 14) that is neither exactly 1 nor results in a penalty function below the truncation threshold, $h_{\text {thre }}$. This allows a prescreening of particles, and reduces the size of system from $\left(N_{\mathrm{QM}}, N_{\mathrm{MM}}\right)$ to $\left(N_{\mathrm{QM}}^{\mathrm{Imp}}, N_{\mathrm{MM}}^{\mathrm{Imp}}\right)$ based on the upper bounds to $h^{\text {FlexiBLE }}$ in Eqs. 17 and 18.

To see how pre-screening works, consider the specific case of 4 QM particles and 4 MM particles. The denomi- nator of Eq. 16 involves a sum over $h^{\text {FlexiBLE }}$ terms resulting from all possible QM and MM exchanges. However, if every exchange producing a QM label at the eighth particle results in $h^{\text {FlexiBLE }}$ below the threshold, $h_{\text {thre }}$, these terms are truncated, and the only surviving terms all have an MM label at the eighth particle position. Following Eq. 15, every surviving pair function, $g$, involving particle 8 is exactly 1 and does not alter $h^{\text {FlexiBLE }}$, thus particle 8 can be excluded entirely in the construction of $h^{\text {FlexiBLE }}$, and $N_{\mathrm{MM}}^{\mathrm{Imp}}$ can be reduced by one.

To efficiently pre-screen particle indices, we seek an upper bound to $h^{\text {FlexiBLE }}$ following any exchange pattern that leaves the outermost QM label at the particle position under consideration. In the $4 \mathrm{QM}, 4 \mathrm{MM}$ example, it is clear that the largest $h^{\text {FlexiBLE }}$ value is $h[\mathrm{AAABBBBA}]$ (using the notation introduced in Appendix B). Any further exchanges (indicated below with "X" labels) will lead to a smaller $h$, according to the tree hierarchy relation in Appendix B. Therefore, starting from perfect $\mathrm{QM} / \mathrm{MM}$ separation, the single exchange between the outermost QM label (particle 4) and the eighth particle label (MM) serves as the upper bound for pre-screening:

$$
\begin{gathered}
h[\mathrm{XXXXXXXA]} \leq h[\mathrm{AAABBBBA}]= \\
g_{84} \times g_{85} \times g_{86} \times g_{87}=h_{8}^{\mathrm{MM} \text { bound }} .
\end{gathered}
$$


Generalizing to arbitrary $N_{\mathrm{QM}}$ and $N_{\mathrm{MM}}$, the $\mathrm{MM}$ bound for particle $q^{\prime}$ is:

$$
\begin{aligned}
& h[\underbrace{\mathrm{X} \cdots \mathrm{X}}_{q^{\prime}-1 \geq N_{\mathrm{QM}}} \mathrm{AB} \cdots \mathrm{B}] \leq h[\underbrace{\mathrm{A} \cdots \mathrm{A}}_{N_{\mathrm{QM}}-1} \underbrace{\mathrm{B} \cdots \mathrm{B}}_{q^{\prime}-N_{\mathrm{QM}}-1} \mathrm{AB} \cdots \mathrm{B}]= \\
& \prod_{i=N_{\mathrm{QM}}}^{q^{\prime}-1} g_{q^{\prime}, i}=h_{q^{\prime}}^{\mathrm{MM}} \text { bound } .
\end{aligned}
$$

This proves Eq. 18. The proof of Eq. 17 follows similarly.

\section{REFERENCES}

${ }^{1}$ A. Warshel and M. Levitt, J. Mol. Biol. 103, 227 (1976).

${ }^{2} \mathrm{~J}$. Gao, "Methods and applications of combined quantum mechanical and molecular mechanical potentials," in Reviews in Computational Chemistry (John Wiley Sons, Ltd, 1996) pp. 119-185.

${ }^{3}$ T. Kerdcharoen and K. Morokuma, Chem. Phys. Lett. 355, 257 (2002).

${ }^{4}$ H. Lin and D. G. Truhlar, Theor. Chem. Acc. 117, 185 (2006).

${ }^{5}$ S. C. L. Kamerlin, M. Haranczyk, and A. Warshel, J. Phys. Chem. B 113, 1253 (2009).

${ }^{6}$ H. M. Senn and W. Thiel, Angew. Chem. Int. Ed. 48, 1198 (2009).

${ }^{7}$ A. Heyden, H. Lin, and D. G. Truhlar, J. Phys. Chem. B 111, 2231 (2007)

${ }^{8}$ R. E. Bulo, B. Ensing, J. Sikkema, and L. Visscher, J. Chem. Theory Comput. 5, 2212 (2009).

${ }^{9}$ N. Bernstein, C. Várnai, I. Solt, S. A. Winfield, M. C. Payne, I. Simon, M. Fuxreiter, and G. Csányi, Phys. Chem. Chem. Phys. 14, 646 (2012).

${ }^{10}$ C. N. Rowley and B. Roux, J. Chem. Theory Comput. 8, 3526 (2012).

${ }^{11}$ N. Takenaka, Y. Kitamura, Y. Koyano, and M. Nagaoka, Chem. Phys. Lett. 524, 56 (2012).

${ }^{12}$ M. Shiga and M. Masia, J. Chem. Phys. 139, 044120 (2013).

${ }^{13}$ M. Shiga and M. Masia, J. Chem. Phys. 139, 144103 (2013).

${ }^{14}$ M. P. Waller, S. Kumbhar, and J. Yang, ChemPhysChem 15, 3218 (2014).

${ }^{15}$ A. Warshel, Angew. Chem. Int. Ed. 53, 10020 (2014).

${ }^{16}$ H. C. Watanabe, T. Kubař, and M. Elstner, J. Chem. Theory Comput. 10, 4242 (2014).

${ }^{17}$ X. Lu, D. Fang, S. Ito, Y. Okamoto, V. Ovchinnikov, and Q. Cui, Mol. Sim. 42, 1056 (2016).

${ }^{18}$ M. J. Field, J. Chem. Theory Comput. 13, 2342 (2017).

${ }^{19}$ H. Takahashi, H. Kambe, and A. Morita, J. Chem. Phys. 148, 134119 (2018).

${ }^{20}$ U. Morzan, D. J. Alonso de Armiño, N. Foglia, F. Ramírez, M. C. González Lebrero, D. A. Scherlis, and D. A. Estrin, Chem. Rev. 118, 4071 (2018).

${ }^{21}$ H. C. Watanabe and Q. Cui, J. Chem. Theory Comput. 15, 3917 (2019).

${ }^{22}$ F. Libisch, C. Huang, and E. A. Carter, Acc. Chem. Res. 47, 2768 (2014).

${ }^{23}$ C. R. Jacob and J. Neugebauer, Wiley Interdiscip. Rev. Comput. Mol. Sci. 4, 325 (2014).

${ }^{24}$ M. E. Fornace, J. Lee, K. Miyamoto, F. R. Manby, and T. F. Miller, J. Chem. Theory Comput. 11, 568 (2015).

${ }^{25}$ V. V. Rybkin, J. Chem. Theory Comput. 17, 3995 (2021).

${ }^{26}$ P. Sherwood, A. H. de Vries, M. F. Guest, G. Schreckenbach, C. R. A. Catlow, S. A. French, A. A. Sokol, S. T. Bromley, W. Thiel, A. J. Turner, S. Billeter, F. Terstegen, S. Thiel,
J. Kendrick, S. C. Rogers, J. Casci, M. Watson, F. King, E. Karlsen, M. Sjøvoll, A. Fahmi, A. Schäfer, and C. Lennartz, J. Mol. Struct. THEOCHEM 632, 1 (2003).

${ }^{27}$ Y. Zhang, Theor. Chem. Acc. 116, 43 (2006).

${ }^{28} \mathrm{H}$. Hu and W. Yang, J. Mol. Struct. THEOCHEM 898, 17 (2009).

${ }^{29}$ E. M. Sproviero, M. B. Newcomer, J. A. Gascón, E. R. Batista, G. W. Brudvig, and V. S. Batista, Photosynth. Res. 102, 455 (2009).

${ }^{30}$ S. Difley, L.-P. Wang, S. Yeganeh, S. R. Yost, and T. V. Voorhis, Acc. Chem. Res. 43, 995 (2010).

${ }^{31}$ E. Brunk and U. Rothlisberger, Chem. Rev. 115, 6217 (2015).

${ }^{32}$ T. J. Zuehlsdorff and C. M. Isborn, Int. J. Quantum Chem. 119, e25719 (2019).

${ }^{33}$ M. S. Gordon, D. G. Fedorov, S. R. Pruitt, and L. V. Slipchenko, Chem. Rev. 112, 632 (2012).

${ }^{34}$ M. S. Gordon, Q. A. Smith, P. Xu, and L. V. Slipchenko, Annu. Rev. Phys. Chem. 64, 553 (2013).

${ }^{35}$ K. Bolton, W. L. Hase, and C. Doubleday, J. Phys. Chem. B 103, 3691 (1999).

${ }^{36}$ C. M. Isborn, A. W. Götz, M. A. Clark, R. C. Walker, and T. J. Martínez, J. Chem. Theory Comput. 8, 5092 (2012).

${ }^{37}$ J. R. Casey, A. Kahros, and B. J. Schwartz, J. Phys. Chem. B 117, 14173 (2013).

${ }^{38}$ L. Turi and P. J. Rossky, Chem. Rev. 112, 5641 (2012).

${ }^{39}$ J. M. Herbert, Phys. Chem. Chem. Phys. 21, 20538 (2019).

${ }^{40}$ X. Chen and S. E. Bradforth, Annu. Rev. Phys. Chem. 59, 203 (2008).

${ }^{41}$ E. Lambros, F. Lipparini, G. A. Cisneros, and F. Paesani, J. Chem. Theory Comput. 16, 7462 (2020).

${ }^{42}$ M. Shiga and M. Masia, Mol. Sim. 41, 827 (2015).

${ }^{43}$ E. J. Hart and J. W. Boag, J. Am. Chem. Soc. 84, 4090 (1962).

${ }^{44}$ L. Turi and D. Borgis, J. Chem. Phys. 117, 6186 (2002).

${ }^{45}$ Z. Shen, S. Peng, and W. J. Glover, J. Chem. Phys. X (2021).

${ }^{46}$ W. J. Glover and B. J. Schwartz, J. Chem. Theory Comput. 16, $1263(2020)$.

${ }^{47}$ D. Case, I. Ben-Shalom, S. R. Brozell, D. S. Cerutti, T. Cheatham, V. W. D. Cruzeiro, T. Darden, R. Duke, D. Ghoreishi, M. Gilson, H. Gohlke, A. Götz, D. Greene, R. Harris, N. Homeyer, Y. Huang, S. Izadi, A. Kovalenko, T. Kurtzman, and P. A. Kollman, Amber 2018 (University of California, San Francisco, 2018).

${ }^{48}$ W. J. Glover and B. J. Schwartz, J. Chem. Theory Comput. 12, 5117 (2016)

${ }^{49}$ W. J. Glover, R. E. Larsen, and B. J. Schwartz, J. Chem. Phys. 129, 164505 (2008).

${ }^{50}$ W. J. Glover, J. R. Casey, and B. J. Schwartz, J. Chem. Theory Comput. 10, 4661 (2014).

${ }^{51}$ G. Bussi, D. Donadio, and M. Parrinello, J. Chem. Phys. 126, 014101 (2007).

${ }^{52}$ W. C. Swope, H. C. Andersen, P. H. Berens, and K. R. Wilson, J. Chem. Phys. 76, 637 (1982).

${ }^{53}$ K. Neymeyr, Linear Algebra Its Appl. 322, 61 (2001).

${ }^{54}$ V. Hernandez, J. E. Roman, and V. Vidal, ACM Trans. Math. Software 31, 351 (2005).

${ }^{55}$ R. P. Feynman, Phys. Rev. 56, 340 (1939).

${ }^{56}$ G. W. Stewart, SIAM J. Matrix Anal. Appl. 23, 601 (2002).

${ }^{57}$ L. Turi, G. Hantal, P. J. Rossky, and D. Borgis, J. Chem. Phys. 131, 024119 (2009).

${ }^{58}$ M. A. Thompson and G. K. Schenter, J. Phys. Chem. 99, 6374 (1995).

${ }^{59}$ J. Gao, J. Comp. Chem. 18, 1061 (1997).

${ }^{60} \mathrm{~J}$. Kongsted and B. Mennucci, J. Phys. Chem. A 111, 9890 (2007).

${ }^{61}$ M. A. Hagras and W. J. Glover, J. Chem. Theory Comput. 14, 2137 (2018).

${ }^{62}$ Consider QM/MM strings according to the above sequences. $b_{1}<$ $a_{N_{\mathrm{QM}}^{\mathrm{imp}}}$ implies that there exists some MM index on the left of some QM index. Then between these indices there must exist a consecutive MM-QM pair, otherwise the string can only be of MM indices, which is unphysical. 